\title{
Enabling Reuse of Process Models through the Detection of Similar Process Parts
}

\author{
Fabian Pittke ${ }^{1,3}$, Henrik Leopold ${ }^{1}$, Jan Mendling $^{2}$, and Gerrit Tamm ${ }^{3}$ \\ 1 Humboldt-Universität zu Berlin, Unter den Linden 6, 10099 Berlin, Germany \\ henrik.leopold@wiwi.hu-berlin.de \\ 2 WU Vienna, Augasse 2-6, A-1090 Vienna, Austria \\ jan.mendling@wu.ac.at \\ 3 SRH University Berlin, Ernst-Reuter-Platz 10, 10587 Berlin, Germany \\ fabian.pittke@srh-uni-berlin.de gerrit.tamm@srh-uni-berlin.de
}

\begin{abstract}
Many companies use business process modeling to support various improvements initiatives leading to an increasing number of process models. Typically, these models are stored in a collection containing several hundreds of process models. In many cases, process models are overlapping, although parts could be easily reused saving costs and efforts. Different labeling styles and evolving process models complicate the detection of reusable model parts. In this paper, we propose a novel approach for the detection of equivalent and similar process model parts that exploits semantic comparison of activity labels and behavioral comparison of control flow. We evaluate our approach on the SAP Reference Model, a collection with 604 process models. The evaluation reveals insights for the thresholds of semantic and behavioral similarity of process models as well as their influence for similar process part detection. Hence, we identify five candidate groups with specific similarity properties that contain reoccurring process parts.
\end{abstract}

\section{Introduction}

Due to the increasing popularity of business process modeling many companies face a steadily increasing amount of process models. In some cases, such process model collections range up to thousands of process models 1. As a result, these companies struggle with the effective maintenance of their process model collections [2.

A corresponding problem of growing model collections is the increasing overlap across process models. Hence, the implementation of consistent changes becomes more and more challenging and cost intensive. Moreover, size and number of overlapping process models is often larger than it necessarily had to be. Reoccurring parts could be easily extracted in form of separate process models that reduce the complexity of the models themselves and of the overall collection. Recent research addressed this problem by detecting clones in process model repositories [3. Although the detection of clones is undoubtedly a very useful step, it is not complete. Moreover, most approaches only focus on structural 
aspects and disregard semantic aspects. As a consequence, semantically equal process model parts which are not exact matches remain undetected and impede reuse.

There are at least two issues that complicate non-exact reuse. First, the labeling of process model elements in practice is heterogeneous and modelers use different labeling patterns to express the same semantics [4. Thus, equivalent process parts with differently labeled elements would not be recognized as the same. Second, minor changes, for instance the insertion of an additional activity or the usage of other words, impede the identification of similar process parts. In this paper, we address this problem by introducing an approach for the identification of semantically equivalent and similar process parts in process model repositories. Our approach exploits the semantic comparison of activity labels and the behavioral comparison of control flow aspects. To demonstrate the applicability of our approach, we conduct an evaluation with the SAP Reference Model. We also provide insights into the sensitivity of the similarity thresholds for semantic and behavioral similarity and their influence for process part detection. While our approach detects clones for high similarity thresholds, it still detects meaningful candidates, when loosen the clone requirement.

The remainder of this paper is structured as follows. Section 2 illustrates the motivation of our work. Section 3 defines our approach for detecting similar process model parts. Section 4 presents the results from our empirical evaluation with the SAP Reference Model. Section 5 discusses related work before Section 6 concludes the paper and gives an outlook on future research.

\section{Problem Illustration}

The main challenge associated with the identification of similar process parts is given by appropriately covering semantic aspects. While the identification of clones can be accomplished on a structural level [3], this is not possible for process parts deviating in labeling style and behavior.

Figure 1 shows a typical example of two similar process models from the SAP Reference Model. Thereby, functions with a bold line represent activities which are not covered by the other process. Bold font indicates that the function has a corresponding function in the other model, but captures the semantics in a linguistically different way. Considering the models, we observe that both have additional functions and that two functions differ in the label. However, it is also obvious the these models are semantically very close. For instance the label Processing of Shipping Notifications / Confirmations gives the same instruction as the label Shipping Notifications / Confirmation Processing. In this case the two functions simply make use of different label styles resulting in a different position of the action to process [4. While this is a rather syntactical difference, the labels Scheduling Agreement Delivery Schedule and Schedule Line (Schedule Agreement) represent a semantically more complex example.

The comparison of process models is a widely discussed area and many metrics have been defined. Thereby, many metrics build on the process model 


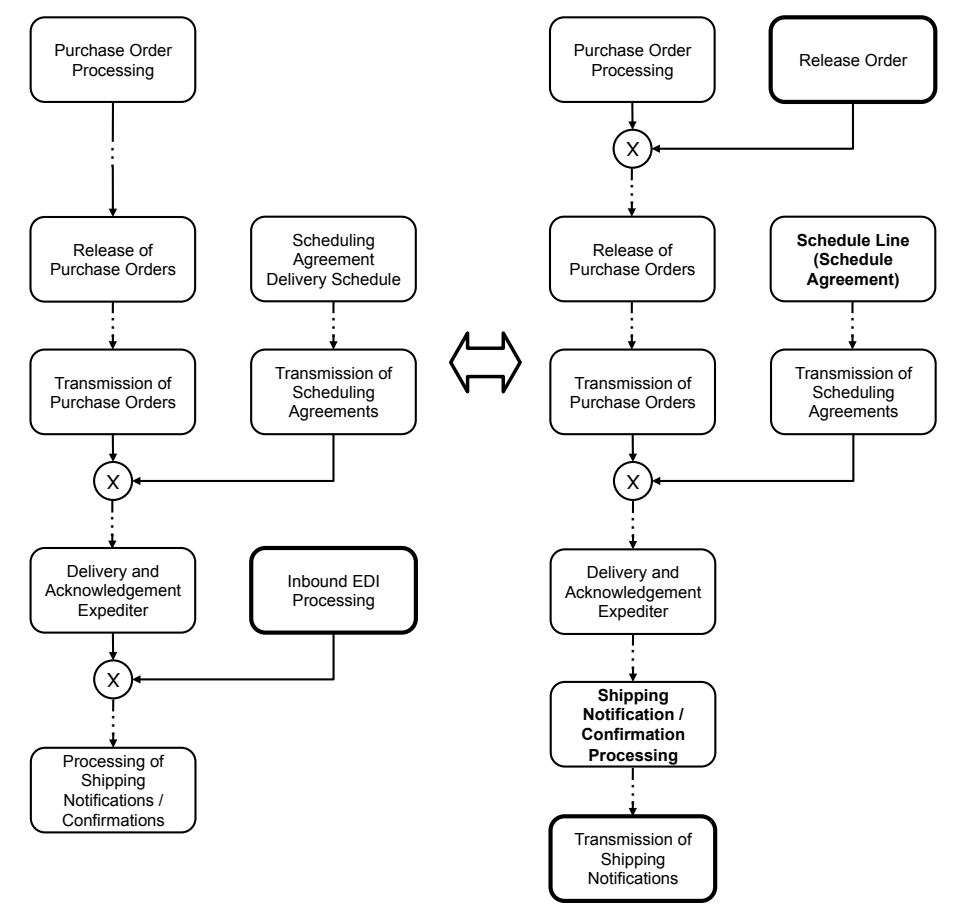

Fig. 1. Almost Completely Similar Process Parts from the SAP Reference Model

structure [5, 6] or the execution semantics [7, 8. However, the calculation of a semantic similarity of process model labels which goes beyond the consideration of synonyms has not been addressed so far. However, because of the potential of similar process parts to increase the reuse in process model collections, we consider this to be an important step.

\section{Approach for the Detection of Similar Process Parts}

This section introduces our three-step approach for the detection of similar process parts. It includes (1) activity label annotation, (2) semantic label similarity calculation, and (3) behavioral similarity calculation.

\subsection{Activity Label Annotation}

In order to accomplish a comparison which goes beyond a simple string comparison we annotate each activity with its semantic components. As pointed out by [9] each activity can be characterized by three components: an action, a business object, on which the action is performed, and an additional information fragment that provides further details if required. As an example, consider the activity Send Contract to Customer which contains the action to send, the business 
object contract and the additional information fragment to customer. To reliably accomplish the annotation of these components, we employ a technique defined in prior work [4. The introduced technique builds on the observation that activities follow regular patterns, so called label styles. The most prominent label style is the verb-object style where the action is captured as an infinitive verb at the beginning of the label. Examples are Notify Customer or Print Document. However, many labels follow actually other styles such as the action-noun style. In these cases the action is not given as a verb but as a noun at the end of the label. Examples are Order Verification or Product Shipment. The given examples demonstrate that knowledge about label styles can be used to annotate activities. Once the label style is identified, the derivation of the components is a straightforward step.

\subsection{Semantic Label Similarity Calculation}

The calculation of the semantic process similarity builds on the annotation of the comprised activities. The fundamental idea is to first calculate the semantic similarity between all activities of the input models and then compute an overall similarity score.

The calculation of the semantic similarity between two activities is accomplished as follows. As a result of the annotation, we can use the semantic components of two given activity labels to compute their semantic closeness. Hence, we consider the similarity between the actions, the business objects and the additional fragments. Thereby, the similarity between two components is given by the closeness of the two concepts in the WordNet taxonomy [10]. This closeness can be calculated using a metric defined by Lin [11.

For calculating this semantic similarity between two activity labels $l_{1}$ and $l_{2}$, we introduce three functions: a component similarity function $\operatorname{sim}_{c}$, a coverage function cov, and a label similarity function $\operatorname{sim}_{l}$ combining the latter two to an overall result.

The function $\operatorname{sim}_{c}$ calculates the semantic closeness of two label components $l_{c_{1}}$ and $l_{c_{2}}$. In general, the result of the Lin measurement is returned. If only one label includes the considered component, the value is set to zero.

$$
\operatorname{sim}_{c}\left(l_{1}, l_{2}\right)= \begin{cases}0 & \text { if } l_{1_{c}}=\emptyset \vee l_{2_{c}}=\emptyset \\ \operatorname{Lin}\left(l_{1_{c}}, l_{2_{c}}\right) & \text { if } l_{1_{c}} \neq \emptyset \wedge l_{2_{c}} \neq \emptyset\end{cases}
$$

The coverage function cov is used to determine the number of components in a label $l$. Assuming that a label always refers to an action, the result of cov ranges from 1 to 3 . Note that the index $a$ in the definition denotes the action, $b o$ the business object and add the additional information fragment.

$$
\operatorname{cov}(l)=\left\{\begin{array}{l}
1 \text { if } l_{a} \neq \emptyset \wedge l_{b o}=\emptyset \wedge l_{\text {add }}=\emptyset \\
2 \text { if } l_{a} \neq \emptyset \wedge\left(l_{b o} \neq \emptyset \vee l_{\text {add }} \neq \emptyset\right) \\
3 \text { if } l_{a} \neq \emptyset \wedge l_{b o} \neq \emptyset \wedge l_{\text {add }} \neq \emptyset
\end{array}\right.
$$

In order to calculate an overall result from the individual similarity results, we introduce the function $\operatorname{sim}_{l}$. It calculates the arithmetic mean of the similarity 
values for action, business object and the additional information fragment. This is accomplished by dividing the sum of $\operatorname{sim}_{a}, \operatorname{sim}_{b o}$ and $\operatorname{sim}_{a d d}$ by the maximum coverage among $l_{1}$ and $l_{2}$. As a result, we obtain the overall similarity score for two given labels.

$$
\operatorname{sim}_{l}\left(l_{1}, l_{2}\right)=\frac{\operatorname{sim}_{a}\left(l_{1}, l_{2}\right)+\operatorname{sim}_{b o}\left(l_{1}, l_{2}\right)+\operatorname{sim}_{a d d}\left(l_{1}, l_{2}\right)}{\underset{l \in\left\{l_{1}, l_{2}\right\}}{\arg \max \operatorname{cov}(l)}}
$$

In order to calculate the similarity for the complete process models, we adapt a metric proposed by [5]. By calculating $\operatorname{sim}_{l}$ for all activity pairs which can be derived from the input models we can identify the best activity pairs based on their $\operatorname{sim}_{l}$ value. Accordingly, we use the relation of these pairs and the overall number of activities in both models to yield an overall process similarity score. Let $M_{s i m_{l}}^{o p t}$ be an optimal equivalence mapping derived from siml. Further $A$ represents the set of activities in a given process models $P$. As a result, we can define the process model similarity metric $\operatorname{sim}_{p}$ models as follows:

$$
\operatorname{sim}_{p}\left(p_{1}, p_{2}\right)=\frac{2 \cdot \sum_{\left(l_{1}, l_{2}\right) \in M_{s i m_{l}}^{\text {opt }}} \operatorname{sim}_{l}\left(l_{1}, l_{2}\right)}{\left|A_{1}\right|+\left|A_{2}\right|}
$$

\subsection{Behavioral Similarity Calculation}

Besides containing similarly labeled activities process parts also require these activities to occur in a similar order. To measure such a control-flow oriented similarity of two process models, we use the concept of behavioral profiles [12] and the respective behavioral metric [8].

Behavioral profiles are an abstract representation of control flow aspects. They capture behavioral characteristics by describing the relation between pairs of activities. The possible relations are grounded in the weak order relation between two activities, which holds when there exists a path from activity $x$ to activity $y$, denoted with $x \succ_{P} y$. With this background, behavioral profiles can be defined as follows. Let $A$ be the set of all activities of a given process model $P$. Each pair $(x, y) \in(A \times A)$ has one of the following relations:

- strict order relation $\rightsquigarrow_{P}$, iff $x \succ_{P} y$ and $y \nsucc_{P} x$.

- exclusiveness relation $+_{P}$, iff $x \nsucc_{P} y$ and $y \nsucc_{P} x$.

- interleaving order relation $\|_{P}$, iff $x \succ_{P} y$ and $y \succ_{P} x$.

Thus, a behavioral profile $B_{P}$ of a process model $P$ is defined as $B_{P}=\left\{\rightsquigarrow_{P}\right.$, $\left.+_{P}, \|_{P}\right\}$. Also note, that for each pair $(x, y)$ in strict order relation also fulfills the inverse strict order relation for $(y, x)$, i.e. $x \rightsquigarrow P y \Leftrightarrow y \rightsquigarrow_{P}^{-1} x$.

The behavioral profile metric is based upon these basic relations of a behavioral profile. Let $B_{P}$ and $B_{Q}$ be two behavioral profiles of the process models $P$ and $Q$. Hence, the behavioral similarity is defined as,

$$
\operatorname{sim}_{b p}\left(B_{P}, B_{Q}\right)=1-\sum_{h} w_{h} \cdot \operatorname{sim}_{h}\left(B_{P}, B_{Q}\right)
$$




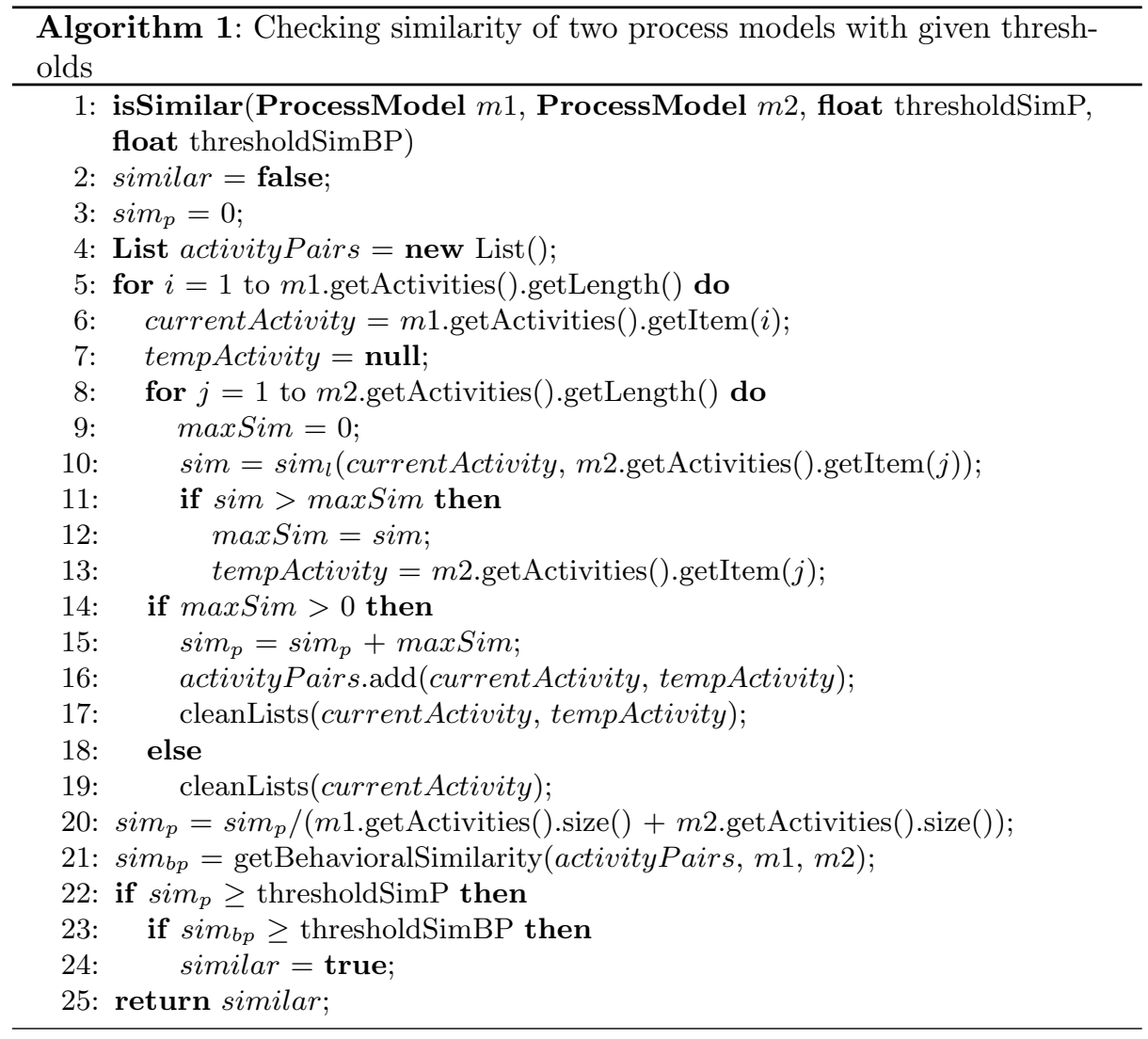

with $h \in\left\{+, \rightsquigarrow,\left\|, \rightsquigarrow^{\prime},\right\|^{\prime}\right\}$ and weighting factors $w_{h} \in \mathbb{R}, 0<w_{h}<1$ such that $\sum_{h} w_{h}=1$.

$\operatorname{sim}_{h}$ refers to the elementary behavioral similarity metrics introduced in 8 .

\subsection{Approach for Detecting Similar Process Parts}

Our approach builds upon the three steps described above. Activity label annotation is performed for each activity label revealing action, business object and the additional fragment. Afterwards, all available process models are compared with each other resulting in a similarity score of $\operatorname{sim}_{p}$. In this step, we additionally identify activities that form a semantic pair. Using these activity pairs we can determine the behavioral similarity of the two process models. If the model pair fulfills certain thresholds for $\operatorname{sim}_{p}$ and $\operatorname{sim}_{b p}$, it is considered to be similar. The approach is formalized in Algorithm 1 taking two process models $m 1$ and $m 2$ as well as thresholds for $\operatorname{sim}_{p}$ and $\operatorname{sim}_{b p}$ as input and returning a boolean score reflecting the similarity and the consistency of the detected process part. 
The algorithm starts with some basic initializations (line 2-3). Afterwards, the algorithm processes all possible pairs of activities from $m 1$ and $m 2$ (line 4 , line 7) as follows. The similarity scores between currentActivity from $m 1$ and the activities from $m 2$ are calculated. If the similarity of currentActivity and the respective activity from $m 2$ is higher than the highest similarity score (maxSim) calculated so far, maxSim is updated by the new similarity score and the respective activity from $m 2$ temporarily stored (lines 10-13). These steps are repeated for all activities from $m 2$. Afterwards, the algorithm checks, whether the maximal calculated similarity is bigger than zero (line 13). If this is the case, the similarity of the input models is increased by the respective similarity (line 15) and the activity pair contributing the similarity score is added to activityPairs, a list that contains all pairs of activities with the highest pairwise similarity score (line 16). Additionally, the activity lists of $m 1$ and $m 2$ are cleaned from the pair activities to prevent one activity occurring in multiple activity pairs (line 17 and line 19). After the calculation of the final model similarity score (line 20), the algorithm proceeds with the calculation of the behavioral similarity using the two process models $m 1$ and $m 2$ as well as the list activityPairs as input (line 21). If $\operatorname{sim}_{p}$ and afterwards $\operatorname{sim}_{b p}$ exceed the two thresholds, two boolean variable similar is set to true (lines 22-24). The algorithm terminates with the output of similar indicating a similarity or not (line 25$)$.

\section{Evaluation}

In this section we evaluate the introduced approach for detecting similar process parts. To this end, we test our technique on the SAP Reference Model, a model collection containing 604 Event-Driven Process Chains [13]. The comprised process models are organized in 29 functional branches, as for instance procurement, sales or financial accounting. In Section 4.1 we present the general results from our test run. In Section 4.2 we discuss the relation between $\operatorname{sim}_{p}$ and $\operatorname{sim}_{b p}$ as well as their influence for the detection of process model parts.

\subsection{General Results}

We conduct a pair-wise comparison of all models in the SAP Reference Model to identify similar processes. Accordingly, we conducted in total $\left(\begin{array}{c}604 \\ 2\end{array}\right)=182.106$ comparisons and computed $\operatorname{sim}_{p}$ and $\operatorname{sim}_{b p}$ for each model pair in the collection. The results are depicted in Figure 2 It illustrates the number of retrieved process model pairs depending on varying thresholds for $\operatorname{sim}_{p}$. Apparently, the more we decrease the threshold for the $\operatorname{sim}_{p}$, the more process model pairs are identified by the algorithm.

We also computed the average $\operatorname{sim}_{b p}$ values for the respective $\operatorname{sim}_{p}$ as illustrated in Figure 3. We observe a proportional relation between the decrease of $\operatorname{sim}_{p}$ and the average $\operatorname{sim}_{b p}$ value, i.e. $\operatorname{sim}_{b p}$ is dependent on $\operatorname{sim}_{p}$. This is the case, because $\operatorname{sim}_{b p}$ requires the correspondences between the elements 
of the two input models that is provided by $\operatorname{sim}_{p}$ [8]. In consequence, weaker correspondences between the model pairs also result in a weaker behavioral similarity.

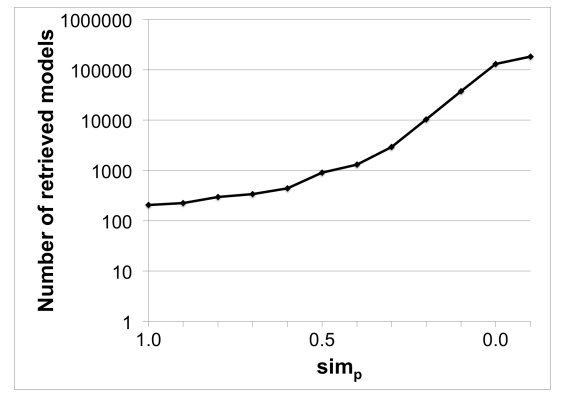

Fig. 2. Retrieved models for $\operatorname{sim}_{p}$ thresholds

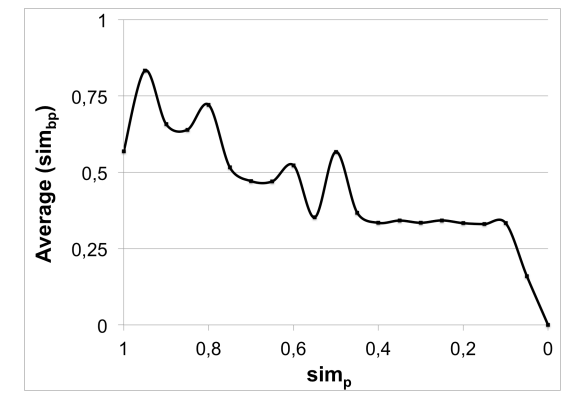

Fig. 3. Avg. $\operatorname{sim}_{b p}$ for thresholds $\operatorname{sim}_{p}$

\subsection{Similarity Thresholds for Detecting Similar Process Parts}

Since different thresholds for the metrics $\operatorname{sim}_{p}$ and $\operatorname{sim}_{b p}$ entail completely different results, we investigated how these metrics should be configured in order to obtain the desired outcome. As illustrated in Figure 2, a step-wise decrease of $\operatorname{sim}_{p}$ threshold leads to an increase of the retrieved model pairs, while it reduces the average score of $\operatorname{sim}_{b p}$. Apparently, $\operatorname{sim}_{p}$ has a significantly stronger influence on the detection of similar process parts than $\operatorname{sim}_{b p}$.

Taking the results of Section 4.1 into consideration, we can identify several candidate groups based on a given threshold for $\operatorname{sim}_{p}$. We summarized all candidate groups in Table 1. The first candidate group amounts to 204 model pairs and contains process models that are perfect clones. Process models of this group have an equal number of activities and equivalent activity labels.

The next candidate group has a $\operatorname{sim}_{p}$ score between 0.6 and 1.0. These models tend to include activity clones as well as semantically similar activities. In general, they differ in the number of activities. The activities themselves are either clones or semantically (very) close activities sharing similar actions and business objects. The example models in Figure 1 represent candidates of this group. For the example $\operatorname{sim}_{p}$ amounts to 0.62 .

Candidate group 3 comprises models with an increasing semantic distance. While the semantic closeness of these models is still meaningful for higher scores in this range, models tend to become more and more distant for lower bound thresholds. Hence, the respective activities tend to either share a similar business object or the same action. Figure 4 provides an example of process models that is on the edge of group 3 and 4 . The value of $\operatorname{sim}_{p}$ amounts to 0.27 and we note only weak semantic relations between the activities. Consider for instance the 
activities Appropriation Request Processing and Process Inquiry. Obviously, both activities share the action process that is performed on a business object leading to the correspondence.

As the largest candidate group 4 covers models with small semantic correspondences where activities only share a similar business object or a similar activity. Normally, human perception would ignore these models to be similar. Group 5 consists of all model pairs with no semantic correspondence scoring zero for $\operatorname{sim}_{p}$.

\begin{tabular}{|l|l|l|l|}
\hline $\begin{array}{l}\text { Group } \\
\text { Number }\end{array}$ & Similarity Tendency & Threshold & Group size \\
\hline \hline 1 & Perfect Model Clones & $\operatorname{sim}_{p}=1.0$ & 204 \\
\hline 2 & Clone or semantically close models & $0.6 \leq \operatorname{sim}_{p}<1.0$ & 237 \\
\hline 3 & Semantically similar to distant models & $0.3 \leq \operatorname{sim}_{p}<0.6$ & 2,481 \\
\hline 4 & Semantically distant models & $0.0<\operatorname{sim}_{p}<0.3$ & 128,240 \\
\hline 5 & Semantically dissimilar models & $\operatorname{sim}_{p}=0.0$ & 50,944 \\
\hline
\end{tabular}

Table 1. Candidate groups of $\operatorname{sim}_{p}$

As already stated above, the decrease of the $\operatorname{sim}_{p}$ threshold corresponds with a decrease of the $\operatorname{sim}_{b p}$ value. We observed a high discrepancy between $\operatorname{sim}_{p}$ and $\operatorname{avg}\left(\operatorname{sim}_{b p}\right)$ for group 1 (about 43\%) and for group 2 (about 22\%). In general, most process models only comprise two of the three used behavioral relations for $\operatorname{sim}_{b p}$, which leads to a smaller score of $\operatorname{sim}_{b p}$. The divergence of candidate group 3 is rather small (8\%), whereas group 4 shows a high discrepancy again (about 65\%). Again, two behavioral relations primarily contribute to $s i m_{b p}$ leading to a relatively high $\operatorname{sim}_{b p}$ compared to $\operatorname{sim}_{p}$. Considering the example from Figure $4 \operatorname{sim}_{b p}$ amounts to 0.42 , which implies a similar control-flow. There is no discrepancy in group 5 , because of the fact that no pair of corresponding activities is found, if $\operatorname{sim}_{p}=0.0$.

Due to this high discrepancies for higher or smaller thresholds for $\operatorname{sim}_{p}$ we conclude that $\operatorname{sim}_{b p}$ is not appropriate for the initial identification of similar process parts. This is supported by the fact that the correspondence of process models which is required for the calculation of $\operatorname{sim}_{b p}$ is strongly dependent on $\operatorname{sim}_{p}$. We conclude that $\operatorname{sim}_{b p}$ is more appropriate to verify the correctness of an identified process part, while $\operatorname{sim}_{p}$ is able to identify meaningful candidates in a given collection. In other words, $\operatorname{sim}_{p}$ imposes a necessary and $\operatorname{sim}_{b p}$ a sufficient condition for process part detection.

\section{Related Work}

The work presented in this paper is related to three major streams of research: process model reuse, process model similarity and process model matching. 


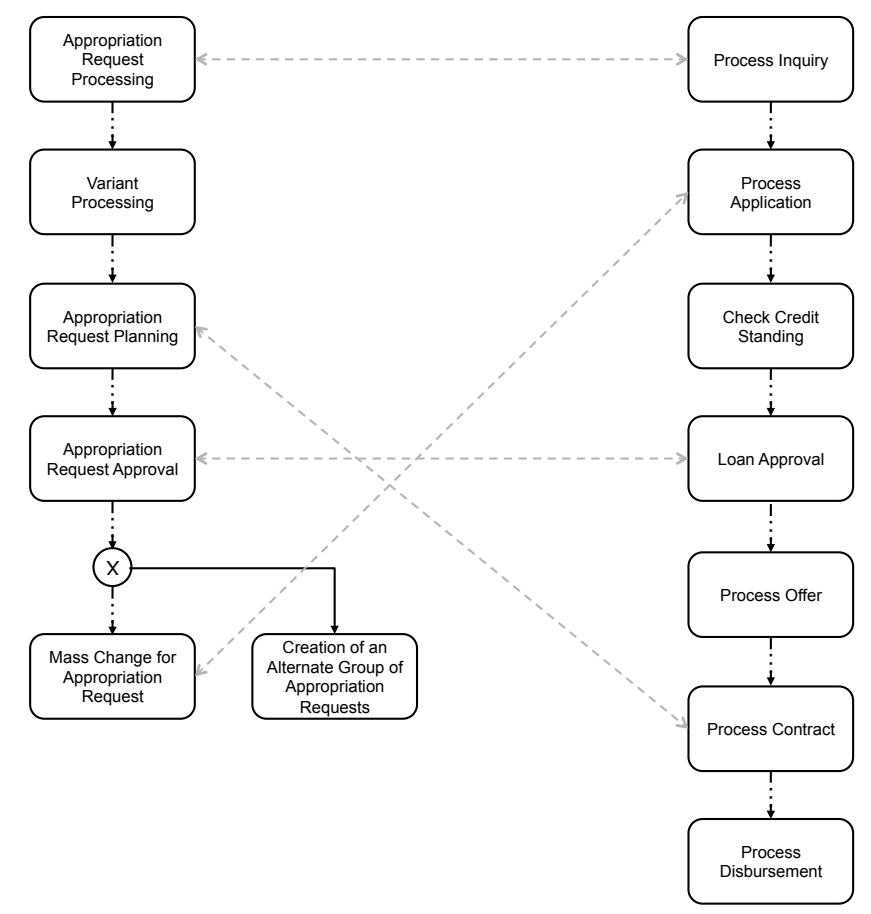

Fig. 4. Semantically distant process models with their corresponding activities

In disciplines such as software engineering reuse has a long tradition [14, 15, 16. Identifying and reusing code fragments and software components does not only save time but also increases the maintainability of the resulting software artifacts. This is line with the Service-Oriented Architecture paradigm, where business functionality is bundled and centrally provided [17, 18. Recently, this potential was also recognized for business process models. 3] proposed an approach for identifying clones in process model repositories. Our approach deviates from this technique, because it does not aim for detecting clones but explicitly targets the identification of similar process parts. Hence, our approach includes techniques for determining the semantic closeness of models based on their control flow and activity labels. Another technique was introduced by [19. The authors propose an approach to support to the design and modeling of workflows by introducing a repository, which can be used for adapting workflow cases.

Techniques for determining the overall similarity of process models have been proposed by different authors. An overview is provided by [5]. Some of these works build on an ontology in order to match the labels [20. Other approaches make use of control-flow based aspects. For instance, [8] use behavioral profiles to determine the similarity of processes. However, this approach assumes that the correspondences between the activities are already given. In general, the vast majority of these approaches focus on structural aspects and do not take semantic 
aspects into account. As this is crucial for the identification of similar process parts, we use a metric which builds on the semantic comparison of element labels.

The alignment or so-called matching of process models is closely connected with the similarity computation. Usually similarity scores are used to identify potential correspondences between two models which are then used as input for the matching technique 21, 22. However, while matchers try to find the best match for a given pair of models, our approach aims for quantifying the semantic similarity between them. Accordingly, a perfect match is not a prerequisite for our approach. In our context it is more important to identify models which have a certain degree of similarity. As a result, the identification of reuse candidates is automatically accomplished.

\section{Conclusion}

In this paper, we have proposed an approach that detects similar parts of process models. We exploit semantic and behavioural similarity aspects and challenged our approach against the SAP Reference Model. Our evaluation proved that the approach is applicable to real-world process models. It also revealed five candidate groups and configurable similarity thresholds enabling process part detection. We concluded that semantic similarity represents a necessary requirement for the detection of process parts, while the behavioral similarity formulates a sufficient condition ensuring correctness and consistency of the identified process parts.

There are several directions of our future work. First, we aim at improving our approach. This especially applies for the identification of model correspondences using semantic similarity techniques. Accordingly, we plan to incorporate more sophisticated matching algorithms in order to obtain a more precise $\operatorname{sim}_{p}$ value. Second, we plan to test our approach on further process model collections, as for instance the BIT process library [23] or the process repository of "Nationale Prozessbibliothek" 11 In addition, we aim for testing our approach in an industrial setting. We think that the resulting feedback will help us to tailor approach to the actual needs of organizations. A third direction for future work is given by combining our approach with other technique facilitating reuse. Particularly, we plan to integrate the approach with automatic identification of services [24].

\section{References}

1. Rosemann, M.: Potential Pitfalls of Process Modeling: Part A. Business Process Management Journal 12(2) (2006) 249-254

2. Reijers, H.A., Mans, R.S., van der Toorn, R.A.: Improved model management with aggregated business process models. Data Knowledge Engineering 68(2) (2009) $221-243$

3. Uba, R., Dumas, M., García-Bañuelos, L., Rosa, M.L.: Clone detection in repositories of business process models. In: BPM. (2011) 248-264

\footnotetext{
${ }^{1}$ www.prozessbibliothek.de
} 
4. Leopold, H., Smirnov, S., Mendling, J.: On the refactoring of activity labels in business process models. Information Systems 37(5) (2012) $443-459$

5. Dijkman, R.M., Dumas, M., van Dongen, B.F., Käärik, R., Mendling, J.: Similarity of Business Process Models: Metrics and Evaluation. Information Systems 36(2) (2011) 498-516

6. Grigori, D., Corrales, J., Bouzeghoub, M., Gater, A.: Ranking BPEL Processes for Service Discovery. IEEE Transactions on Services Computing 3(3) (2010) 178-192

7. Zha, H., Wang, J., Wen, L., Wang, C., Sun, J.: A workflow net similarity measure based on transition adjacency relations. Computers in Industry 61(5) (2010) 463-471

8. Kunze, M., Weidlich, M., Weske, M.: Behavioral similarity - a proper metric. In: Proceedings of the 9th International Conference on Business Process Management (BPM 2011), Springer (2011) 166-181

9. Mendling, J., Reijers, H.A., Recker, J.: Activity Labeling in Process Modeling: Empirical Insights and Recommendations. Information Systems 35(4) (2010) 467-482

10. Miller, G.A.: WordNet: a Lexical Database for English. Communications of the ACM 38(11) (1995) 39-41

11. Lin, D.: An information-theoretic definition of similarity. In: Proc. 15th International Conf. on Machine Learning, Morgan Kaufmann, San Francisco, CA (1998) 296-304

12. Weidlich, M., Mendling, J., Weske, M.: Efficient consistency measurement based on behavioural profiles of process models. IEEE TSE 37(3) (2011) 410-429

13. Keller, G., Teufel, T.: SAP(R) R/3 Process Oriented Implementation: Iterative Process Prototyping. Addison-Wesley (1998)

14. Bellon, S., Koschke, R., Antoniol, G., Krinke, J., Merlo, E.: Comparison and evaluation of clone detection tools. IEEE TSE 33(9) (2007) 577-591

15. Jacobson, I., Griss, M., Jonsson, P.: Software Reuse: Architecture, Process and Organization for Business Success. Volume 43. ACM Press (1997)

16. Heineman, G.T., Councill, W.T.: Component-Based Software Engineering: Putting the Pieces Together. Addison-Wesley Professional (2001)

17. Erl, T.: Service-Oriented Architecture: Concepts, Technology, and Design. Prentice Hall PTR, Upper Saddle River, NJ, USA (2005)

18. Marks, E.A., Bell, M.: Service-Oriented Architecture: A Planning and Implementation Guide for Business and Techonology. John Willey \& Sons Inc. (2006)

19. Madhusudan, T., Zhao, J., Marshall, B.: A case-based reasoning framework for workflow model management. Data and Knowledge Engineering 50(1) (2004) 87 115

20. Ehrig, M., Koschmider, A., Oberweis, A.: Measuring Similarity between Semantic Business Process Models. In: APCCM 2007. Volume 67., Ballarat, Victoria, Australia, Australian Computer Science Communications (2007) 71-80

21. Weidlich, M., Dijkman, R.M., Mendling, J.: The ICoP Framework: Identification of Correspondences between Process Models. In: CAiSE 2010. Volume 6051 of LNCS., Springer (2010) 483-498

22. La Rosa, M., Dumas, M., Uba, R., Dijkman, R.M.: Merging Business Process Models. In: OTM 2010. Volume 6426 of LNCS., Springer (2010) 96-113

23. Fahland, D., Favre, C., Jobstmann, B., Koehler, J., Lohmann, N., Völzer, H., Wolf, K.: Instantaneous soundness checking of industrial business process models. In: BPM 2009. Volume 5701 of LNCS., Springer (2009) 278-293

24. Leopold, H., Mendling, J.: Automatic derivation of service candidates from business process model repositories. In: Business Information Systems. Volume 117 of Lecture Notes in Business Information Processing., Springer (2012) 84-95 